\title{
DETERMINAÇÃO DE ZINCO EM SOLO UTILIZANDO COLORIMETRIA
}

\author{
Karina Fraige, Frank Nelson Crespilho e Maria Olímpia Oliveira Rezende* \\ Instituto de Química de São Carlos, Universidade de São Paulo, CP 780, 13560-970 São Carlos - SP, Brasil
}

Recebido em 25/4/06; aceito em 16/8/06; publicado na web em 22/2/07

\begin{abstract}
DETERMINATION OF ZINC IN SOIL USING COLORIMETRY. In terms of soil fertility, zinc is a micronutrient that is very important in the culture of soy, maize, rice and coffee, because it is a structural and functional component of a great number of enzymes. Therefore, diverse methods have been used to determine zinc in soil. In this work we use colorimetry as a methods of zinc determination in soil using two methodologies of sample digesting, $\mathrm{H}_{2} \mathrm{SO}_{4} / \mathrm{H}_{2} \mathrm{O}_{2}$ and $\mathrm{HNO}_{3} / \mathrm{H}_{2} \mathrm{O}_{2}$. In order to compare the results, atomic absorption spectrometry (AAS) was used. Results show that colorimetric methods can be used due to good sensitivity and reproducibility, since the zinc calibration curve showed good linearity. Comparing colorimetric methods with AAS we observed that the results were equivalent, as proven by the statistical values of the F and $\mathrm{t}$ of Student tests. Furthermore, both methods of soil digesting can be used, leading to a flexible methodology of low-cost for routine zinc analyses in soil.
\end{abstract}

Keywords: zinc determination; colorimetry; micronutrient in soil.

\section{INTRODUÇÃO}

O solo tem um papel de fundamental importância nos ciclos da natureza, participando, direta ou indiretamente, da maioria das atividades que ocorrem no planeta. É fonte de alimentos necessários à vida, constituindo-se em uma parte importante da biosfera para os seres humanos, animais e organismos terrestres. A matéria orgânica do solo rege vários processos e interfere, quer nos processos geoquímicos, quer nas propriedades físicas e químicas dos so$\operatorname{los}^{1}$, sendo considerada a principal fonte de nitrogênio para as plantas e fornecedora de elementos como fósforo, enxofre e vários micronutrientes ${ }^{2}$, entre eles, alguns metais. Esses últimos, por sua vez, entram na cadeia alimentar em concentrações muito baixas, motivo pelo qual são denominados micronutrientes, como é o caso do zinco, manganês, cobalto e ferro. A maioria desses elementos compõe importantes ciclos enzimáticos nas plantas, mas tornamse tóxicos e perigosos quando ultrapassam determinadas concentrações-limite ${ }^{3,4}$. Com o intuito de aumentar a produtividade reduzindo os custos da produção agrícola, a incorporação de micronutrientes no solo tornou-se uma das práticas mais rotineiras e extremamente importante para a economia brasileira ${ }^{5}$.

O zinco, cuja abundância nos solos ${ }^{6}$ está entre 25 e $68 \mathrm{mg} \mathrm{L}^{-1}$, é, do ponto de vista da fertilidade do solo, essencial a plantas e animais, pois tem ação como ativador, ou componente estrutural ou funcional de um grande número de enzimas? ${ }^{7}$ É importante no cultivo de soja, milho, arroz e café, participando da síntese do aminoácido triptofano, precursor do AIA (ácido indol acético), um hormônio do crescimento. Portanto, plantas deficientes em zinco são menores, raquíticas e com internódios curtos, com cloroses internervais e folhas lanceoladas 5 . No entanto, seu excesso pode causar atraso no crescimento e raízes parecidas com arame farpa$\mathrm{do}^{3,8}$. Esse metal pode existir no solo na forma de minerais primários ou íons $\mathrm{Zn}^{2+}$ adsorvidos às partículas mais finas, sendo esta última a forma disponível para as plantas. $\mathrm{O}$ aumento do $\mathrm{pH}$ ou a presença de ânions que levam o zinco a precipitar podem reduzir sua disponibilidade para as plantas no solo9. A deficiência de zinco é comum em áreas de cultivo de trigo em todo o mundo, particular-

*e-mail: mrezende@iqsc.usp.br mente em regiões áridas e semi-áridas ${ }^{10-12}$, e tem ocorrido em uma ampla variedade de solos, agravando-se com o cultivo intensivo ao longo do tempo em países como Austrália, Brasil, China, Colômbia, Índia, Peru e outros do continente asiático ${ }^{13}$. A deficiência de zinco contribui para a diminuição da qualidade nutricional dos grãos e contribui para problemas de saúde em seres humanos, principalmente em países onde os cereais são a principal fonte de alimen$\operatorname{tos}^{11,12}$. No Brasil, a deficiência de zinco é a mais comum entre os micronutrientes, principalmente em solos sob o cerrado e solos arenosos. A carência desse micronutriente acarreta sérios distúrbios ao metabolismo vegetal, os quais se refletem na redução do rendimento de culturas temporárias e perenes ${ }^{13}$. Observa-se preocupação com a deficiência de zinco no estado do Rio Grande do Sul, onde $18,86 \%$ dos solos hidromórficos são ocupados com a cultura do arroz irrigado, sendo a cultura que apresenta maior nível tecnológico e potencial de expansão ${ }^{13}$. Atualmente a Embrapa (Empresa Brasileira de Pesquisa Agropecuária) está envolvida no Programa de Biofortificação de Produtos Agrícolas para Melhor Nutrição Humana, o qual pretende desenvolver, por ex., culturas de feijão e milho com altos teores de zinco, o que poderia diminuir o problema da desnutrição no país, além da deficiência desse nutriente ser responsável por perda da capacidade de trabalho, anemia e problemas no sistema imunológico ${ }^{14}$.

Dentre as principais técnicas e métodos para determinação de zinco no solo, destaca-se, segundo o método 3050B proposto pela EPA ("Environmental Protection Agency"), a digestão de amostra com $\mathrm{HNO}_{3} / \mathrm{H}_{2} \mathrm{O}_{2}$ para análise por espectrometria de emissão atômica por plasma acoplado indutivamente (ICP OES) ou espectrometria de absorção atômica de chama (FLAA) ${ }^{15}$. O método utilizando ICP OES determina elementos traço, incluindo metais, em solução, sendo que todas as matrizes, exceto amostras de águas subterrâneas filtradas, requerem digestão antes da análise ${ }^{17}$. Os limites de detecção, sensibilidade e faixas de concentração linear variam com o comprimento de onda, espectrômetro, matriz e condições de operação, e segundo o método 6010B proposto pela EPA, o limite de detecção instrumental estimado ${ }^{17}$ é de $0,0012 \mathrm{mg} \mathrm{L}^{-1}$. Este método possui a vantagem de realizar determinações multielementares ${ }^{17}$. O método de determinação de metais por espectrometria de absorção atômica (EAA) é simples, rápido e aplicável a um grande número de íons 
metálicos, desde que estejam em solução ou solubilizados por alguma forma de processamento da amostra ${ }^{16}$. Os limites de detecção, sensibilidade e faixas de concentração linear variam com as matrizes e os modelos de espectrômetros e, segundo o método 7000A, proposto pela EPA, o limite de detecção ${ }^{16}$ estimado é de $0,005 \mathrm{mg} \mathrm{L}^{-1}$. Em ambos os métodos, para a digestão utiliza-se aproximadamente $1 \mathrm{~g}$ de amostra, em peso seco, com repetidas adições de $\mathrm{HNO}_{3} \mathrm{e}$ $\mathrm{H}_{2} \mathrm{O}_{2}$. Adiciona-se $\mathrm{HCl}$ à amostra digerida inicialmente e a mistura é mantida sob refluxo. Para aumentar a solubilidade de alguns metais, o digerido é filtrado e o papel de filtro e os resíduos são enxaguados com $\mathrm{HCl}$ quente e água quente, retornando ao frasco de digestão, mantido sob refluxo com $\mathrm{HCl}$ adicional e filtrado novamente. A amostra digerida é então diluída para um volume final de $100 \mathrm{~mL}^{15}$.

A utilização de métodos colorimétricos tem se revelado de grande importância na detecção de zinco e é citada na literatura ${ }^{18,19}$.

$\mathrm{O}$ presente trabalho teve por objetivo desenvolver, comparar e validar a determinação de zinco em matrizes de solo por espectrofotometria na região visível (colorimetria), comparando os resultados aos obtidos por EAA, uma técnica padrão para esse caso. O método utilizado por colorimetria é denominado método Zincon ${ }^{\circledR}$ e consiste na complexação de zinco e outros metais com cianeto, sendo que a liberação seletiva do zinco ocorre com a adição de cicloexanona ao sistema. O zinco então reage com o indicador zincon (2-carboxi-2'-hidroxi-5' -sulfoformazil benzeno) para formar espécies coloridas, cujas intensidades são proporcionais à quantidade de zinco presente ${ }^{6,20}$. Também, avaliaram-se dois métodos para digestão das amostras, um deles utilizando a mistura $\mathrm{H}_{2} \mathrm{SO}_{4} /$ $\mathrm{H}_{2} \mathrm{O}_{2}$ e outro, a mistura $\mathrm{HNO}_{3} / \mathrm{H}_{2} \mathrm{O}_{2}$ em equipamento de digestão Digesdahl ${ }^{\circledR} \mathrm{Hach}^{21}$

\section{PARTE EXPERIMENTAL}

\section{Digestão ácida das amostras}

A amostra de solo utilizada como matriz de estudo foi coletada em uma fazenda na região periférica da cidade de São Carlos-SP, e é classificada como latossolo vermelho, um tipo de solo de coloração vermelha, rico em óxidos de ferro ${ }^{22}$. Para a digestão das amostras utilizou-se o aparelho Digesdahl ${ }^{\circledR}$ Hach, modelo 23130-20,21 , que digere uma ampla variedade de amostras, tais como produtos alimentícios, plantas, fertilizantes, solos, bebidas e óleos, para posterior determinação de nitrogênio total Kjeldahl, minerais e nutrientes ${ }^{21}$. Esse aparelho constitui-se por um balão de digestão, dentro do qual são colocados a amostra e o ácido, e ao qual são acoplados uma coluna de fracionamento e um funil capilar, a partir do qual $\mathrm{H}_{2} \mathrm{O}_{2}$ é adicionado para decomposição completa da amostra. Esse sistema encontra-se sobre uma chapa de aquecimento e possui uma placa de proteção ao seu redor ${ }^{21}$.

A digestão das amostras foi realizada em triplicata e um branco foi preparado a partir de cada ácido, seguindo o mesmo procedimento experimental. Foram avaliadas duas metodologias: digestão com ácido nítrico concentrado e peróxido de hidrogênio 50\%, e digestão com ácido sulfúrico concentrado e peróxido de hidrogênio $50 \%$, sendo todos os reagentes da marca Synth. Pesou-se aproximadamente $0,5000 \mathrm{~g}$ de solo e adicionaram-se 5,0 $\mathrm{mL}$ de ácido sulfúrico concentrado em um balão de digestão Hach de $100 \mathrm{~mL}$, ajustando a temperatura para $440{ }^{\circ} \mathrm{C}$. Após o início da ebulição do ácido sulfúrico deixou-se o sistema em aquecimento por $10 \mathrm{~min}$ e adicionou-se 10,0 mL de peróxido de hidrogênio, aquecendo o sistema por mais $5 \mathrm{~min}$. Em seguida, removeu-se o balão do aquecedor, deixou-se o conteúdo resfriar à temperatura ambiente e completou-se o volume para $100 \mathrm{~mL}$ em um balão volumétrico previamente calibrado. $\mathrm{O}$ mesmo procedimento foi realizado para as amostras digeridas com ácido nítrico, sendo que a quantidade deste foi de $10 \mathrm{~mL}$, temperatura de $200{ }^{\circ} \mathrm{C}$ e volume de peróxido de hidrogênio, de $15 \mathrm{~mL}$.

\section{Colorimetria}

A partir das soluções das amostras resultantes da digestão, utilizou-se para ajuste de $\mathrm{pH}$ soluções de $\mathrm{KOH} 8$ e $0,5 \mathrm{~mol} \mathrm{~L}^{-1}$. Todas as soluções preparadas foram colocadas em recipientes de vidro com tampa de rosca e, quando necessário, armazenadas na geladeira a $5{ }^{\circ} \mathrm{C}$. Para limpeza da vidraria utilizaram-se, respectivamente, detergente alcalino (Extran $®$ ), água destilada, ácido nítrico $30 \%$ (v/v) e água destilada novamente.

Para a construção da curva analítica no espectrofotômetro UVVis foram utilizadas soluções padrão de concentração 0,$0 ; 0,2 ; 0,4$; 0,8 e 1,5 $\mathrm{mg} \mathrm{L}^{-1}$ de zinco (II) (a partir de Zn metálico 99,9\% da Merck), preparadas a partir de uma solução estoque de $1000 \mathrm{mg} \mathrm{L}^{-1}$ por dissolução do metal em ácido nítrico concentrado a quente e posterior preenchimento do balão volumétrico com água desionizada $^{6}$. As análises foram realizadas seguindo a metodologia Zincon ${ }^{\circledR}$, que utiliza comprimento de onda em $620 \mathrm{~nm}^{20}$, em espectrofotômetro de UV-Vis da marca Hach DR 2500. Essa metodologia descreve que o $\mathrm{pH}$ das soluções analisadas deve ser ajustado entre 4 e 5 para que não ocorra a precipitação de sais de zinco. Em seguida, adiciona-se a $20 \mathrm{~mL}$ da amostra o reagente Zinco Ver $5^{20}$, após o qual a amostra fica alaranjada pela complexação de zinco com cianeto. Este é considerado o branco da amostra, do qual são adicionados $10 \mathrm{~mL}$ em uma cubeta. Após a realização dessa etapa, adicionou-se $0,5 \mathrm{~mL}$ de cicloexanona, mistura esta que foi mantida sob agitação durante $30 \mathrm{~s}$, considerada a amostra preparada. Após 3 min o branco é colocado no compartimento do aparelho, que é zerado, colocando-se em seguida a cubeta com a amostra para leitura dos resultados. Os resultados foram substituídos na curva de calibração previamente construída, determinandose os valores de concentração do metal.

Os resultados obtidos pelo método Zincon ${ }^{\circledR}$ foram comparados com a EAA, realizada em aparelho Hitachi, modelo Z-8100, com polarizador Zeeman, chama de ar/acetileno. Para construção da curva analítica no espectrômetro de absorção atômica foram utilizadas soluções padrão preparadas pelo operador do equipamento, nas seguintes concentrações: 0,$00 ; 0,10 ; 0,20 ; 0,40$ e $0,80 \mathrm{mg} \mathrm{L}^{-1}$ de zinco, diluídas a partir da solução estoque. As condições experimentais utilizadas na determinação dos metais foram comprimento de onda $213,9 \mathrm{~nm}$, corrente da lâmpada $10 \mathrm{~mA}$, fenda de $1,3 \mathrm{~nm}$ e fluxo de combustível de $1,5 \mathrm{~L} \mathrm{~min}^{-1}$.

As soluções contendo os resíduos provenientes do desenvolvimento deste trabalho foram encaminhadas ao Laboratório de Resíduos Químicos (LRQ) do Campus USP de São Carlos, para tratamento e recuperação ${ }^{23,24}$.

\section{RESULTADOS E DISCUSSÃO}

\section{Comparação entre os métodos de digestão ácida}

Inicialmente realizou-se uma varredura da amostra por EAA para identificar alguns metais, principalmente alumínio, cádmio, ferro e níquel, que poderiam agir como interferentes na determinação de zinco por colorimetria ${ }^{6,20}$. Cádmio e níquel não foram detectados, mas a concentração de ferro encontrada na amostra foi alta, correspondendo a $(31,2 \pm 0,2) \mathrm{mg} \mathrm{L}^{-1}$ para digestão com $\mathrm{H}_{2} \mathrm{SO}_{4} /$ $\mathrm{H}_{2} \mathrm{O}_{2}$ e $(30,9 \pm 0,1) \mathrm{mg} \mathrm{L}^{-1}$ para digestão com $\mathrm{HNO}_{3} / \mathrm{H}_{2} \mathrm{O}_{2}$, o que se deve à alta quantidade de óxidos de ferro presentes no latossolo vermelho, assim como a concentração de alumínio, correspondendo 
a $(53,7 \pm 5,9) \mathrm{mg} \mathrm{L}^{-1}$ para digestão com $\mathrm{H}_{2} \mathrm{SO}_{4} / \mathrm{H}_{2} \mathrm{O}_{2}$ e $(45,8 \pm 3,1)$ $\mathrm{mg} \mathrm{L}^{-1}$ para digestão com $\mathrm{HNO}_{3} / \mathrm{H}_{2} \mathrm{O}_{2}$ devido à presença dos silicatos de alumínio. No entanto, a quantidade desses metais não foi suficiente para causar interferências na análise de zinco por colorimetria, tendo em vista alguns testes preliminares realizados. Depois de verificada a não influência de outros cátions metálicos nas determinações, construíram-se as curvas analíticas utilizando EAA e colorimetria. A Tabela 1 apresenta as equações de regressão linear e os coeficientes de correlação de cada curva obtida.

Tabela 1. Equações de regressão linear (concentração (y) versus absorbância (x)) e coeficientes de correlação das curvas analíticas obtidas por EAA e colorimetria

\begin{tabular}{lcc}
\hline Método & \multicolumn{1}{c}{ Equação } & Coeficiente de correlação \\
\hline EAA & $\mathrm{y}=0,005+0,0488 \mathrm{x}$ & $\mathrm{r}=0,9999$ \\
Colorimetria & $\mathrm{y}=-0,002+0,6954 \mathrm{x}$ & $\mathrm{r}=1,0000$ \\
\hline
\end{tabular}

Para comparar as metodologias, optou-se neste trabalho por apresentar os resultados em mg do metal por L de solução de solo.

A Tabela 2 apresenta os resultados de concentração de zinco nas amostras, obtidos por EAA, e os limites de detecção e quantificação calculados ${ }^{25}$.

Tabela 2. Concentração, LD e LQ para zinco, em mg $\mathrm{L}^{-1}$, determinados por EAA, com abertura de amostra utilizando $\mathrm{H}_{2} \mathrm{SO}_{4} / \mathrm{H}_{2} \mathrm{O}_{2}$ e $\mathrm{HNO}_{3} / \mathrm{H}_{2} \mathrm{O}_{2}$

\begin{tabular}{lcccc}
\hline Digestão & Amostra & $\begin{array}{c}\text { Concentração } \\
\text { Zn }\left(\mathrm{mg} \mathrm{L}^{-1}\right)\end{array}$ & $\begin{array}{c}\mathrm{LD} \\
\left(\mathrm{mg} \mathrm{L}^{-1}\right)\end{array}$ & $\begin{array}{c}\mathrm{LQ} \\
\left(\mathrm{mg} \mathrm{L}^{-1}\right)\end{array}$ \\
\hline $\mathrm{H}_{2} \mathrm{SO}_{4} / \mathrm{H}_{2} \mathrm{O}_{2}$ & 1 & 0,43 & 0,06 & 0,20 \\
& 2 & 0,48 & & \\
& 3 & 0,43 & & \\
$\mathrm{HNO}_{3} / \mathrm{H}_{2} \mathrm{O}_{2}$ & 4 & 0,38 & 0,04 & 0,13 \\
& 5 & 0,46 & & \\
& 6 & 0,46 & & \\
\hline
\end{tabular}

LQ = Limite de quantificação; LD = Limite de detecção

Os dados apresentados são valores médios de triplicatas obtidos por EAA. A Tabela 3 apresenta os resultados obtidos pelos cálculos de valor médio com desvio da média $(\mu)$, desvio padrão (s) e desvio padrão relativo (RSD) para a determinação de $\mathrm{Zn}$ (II) por EAA.

Tabela 3. Valores de desvio da média ( $\mu$ ), desvio padrão (s) e desvio padrão relativo (RSD) para determinação de zinco por EAA, com abertura de amostra utilizando $\mathrm{H}_{2} \mathrm{SO}_{4} / \mathrm{H}_{2} \mathrm{O}_{2}$ e $\mathrm{HNO}_{3} / \mathrm{H}_{2} \mathrm{O}_{2}$

\begin{tabular}{lcc}
\hline$\mu\left(\mathrm{mg} \mathrm{L}^{-1}\right)$ & $\mathrm{H}_{2} \mathrm{SO}_{4} / \mathrm{H}_{2} \mathrm{O}_{2}$ & $0,45 \pm 0,02$ \\
& $\mathrm{HNO}_{3} / \mathrm{H}_{2} \mathrm{O}_{2}$ & $0,43 \pm 0,05$ \\
$\mathrm{~s}\left(\mathrm{mg} \mathrm{L}^{-1}\right)$ & $\mathrm{H}_{2} \mathrm{SO}_{4} / \mathrm{H}_{2} \mathrm{O}_{2}$ & 0,03 \\
& $\mathrm{HNO}_{3} / \mathrm{H}_{2} \mathrm{O}_{2}$ & 0,04 \\
$\mathrm{RSD}(\%)$ & $\mathrm{H}_{2} \mathrm{SO}_{4} / \mathrm{H}_{2} \mathrm{O}_{2}$ & 6,22 \\
& $\mathrm{HNO}_{3} / \mathrm{H}_{2} \mathrm{O}_{2}$ & 9,07 \\
\hline
\end{tabular}

Por meio das estimativas dos desvios padrão $\left(\mathrm{s}_{\mathrm{H} 2 \mathrm{SO} 4}\right.$ e $\left.\mathrm{s}_{\mathrm{HNO} 3}\right)$ compararam-se as duas metodologias de digestão de amostras por dois testes estatísticos, Teste $\mathrm{F}^{24,25}$ e Teste $\mathrm{t}$ de Student ${ }^{25-27}$.

$\mathrm{O}$ valor de $\mathrm{F}$ obtido para a comparação das duas metodologias de digestão de amostra foi 1,94, sendo o valor tabelado para 9 determinações igual a 3,18. Esse resultado indica que as duas metodo- logias de digestão podem ser utilizadas equivalentemente para determinação de zinco. O valor t obtido foi 1,25 e indica que os resultados são equivalentes. Nesse caso o valor tabelado para 16 graus de liberdade é 2,15 .

Da mesma forma que se compararam as duas metodologias de digestão da amostra de solo por EAA, utilizou-se o método colorimétrico para o mesmo fim. A partir da equação da curva analítica, compararam-se as metodologias e os resultados são apresentados na Tabela 4.

Tabela 4. Concentração, LD e LQ para zinco, em $\mathrm{mg} \mathrm{L}^{-1}$, determinados por colorimetria, com abertura de amostra utilizando $\mathrm{H}_{2} \mathrm{SO}_{4} / \mathrm{H}_{2} \mathrm{O}_{2}$ e $\mathrm{HNO}_{3} / \mathrm{H}_{2} \mathrm{O}_{2}$

\begin{tabular}{lcccc}
\hline Digestão & Amostra & $\begin{array}{c}\text { Concentração } \\
\mathrm{Zn}\left(\mathrm{mg} \mathrm{L}^{-1}\right)\end{array}$ & $\begin{array}{c}\mathrm{LD} \\
\left(\mathrm{mg} \mathrm{L}^{-1}\right)\end{array}$ & $\begin{array}{c}\mathrm{LQ} \\
\left(\mathrm{mg} \mathrm{L}^{-1}\right)\end{array}$ \\
\hline $\mathrm{H}_{2} \mathrm{SO}_{4} / \mathrm{H}_{2} \mathrm{O}_{2}$ & 1 & 0,40 & 0,01 & 0,08 \\
& 2 & 0,44 & & \\
& 3 & 0,48 & & \\
$\mathrm{HNO}_{3} / \mathrm{H}_{2} \mathrm{O}_{2}$ & 4 & 0,40 & 0,01 & 0,08 \\
& 5 & 0,44 & & \\
& 6 & 0,38 & & \\
\hline
\end{tabular}

LQ = Limite de quantificação; LD = Limite de detecção

A Tabela 5 apresenta os resultados obtidos pelos cálculos de valor médio com desvio da média $(\mu)$, desvio padrão (s) e desvio padrão relativo (RSD) para determinação de $\mathrm{Zn}(\mathrm{II})$ por colorimetria.

Tabela 5. Valores de desvio da média ( $\mu$ ), desvio padrão (s) e desvio padrão relativo (RSD) para determinação de zinco por colorimetria, com abertura de amostra utilizando $\mathrm{H}_{2} \mathrm{SO}_{4} / \mathrm{H}_{2} \mathrm{O}_{2}$ e $\mathrm{HNO}_{3} / \mathrm{H}_{2} \mathrm{O}_{2}$

\begin{tabular}{lcc}
\hline$\mu\left(\mathrm{mg} \mathrm{L}^{-1}\right)$ & $\mathrm{H}_{2} \mathrm{SO}_{4} / \mathrm{H}_{2} \mathrm{O}_{2}$ & $0,44 \pm 0,10$ \\
& $\mathrm{HNO}_{3} / \mathrm{H}_{2} \mathrm{O}_{2}$ & $0,41 \pm 0,08$ \\
$\mathrm{~s}\left(\mathrm{mg} \mathrm{L}^{-1}\right)$ & $\mathrm{H}_{2} \mathrm{SO}_{4} / \mathrm{H}_{2} \mathrm{O}_{2}$ & 0,04 \\
& $\mathrm{HNO}_{3} / \mathrm{H}_{2} \mathrm{O}_{2}$ & 0,03 \\
$\operatorname{RSD}(\%)$ & $\mathrm{H}_{2} \mathrm{SO}_{4} / \mathrm{H}_{2} \mathrm{O}_{2}$ & 9,09 \\
& $\mathrm{HNO}_{3} / \mathrm{H}_{2} \mathrm{O}_{2}$ & 7,32 \\
\hline
\end{tabular}

$\mathrm{O}$ resultado de $\mathrm{F}_{\mathrm{c}}$ obtido para zinco foi de 1,78 , sendo o valor tabelado para 3 determinações igual a 19,00, o que indica que ambos os métodos de digestão são equivalentes para a análise colorimétrica.

O resultado obtido para t foi de 1,03 , sendo o valor tabelado para 4 graus de liberdade igual a 2,78, o que indica que ambos os métodos de digestão podem ser utilizados equivalentemente na determinação de zinco.

\section{Comparação entre EAA e Colorimetria}

A Tabela 6 apresenta um resumo dos resultados obtidos por EAA e colorimetria.

Tabela 6. Comparação entre as concentrações de zinco obtidas por colorimetria e por EAA

\begin{tabular}{lcccc}
\hline Método & Abertura & $\begin{array}{c}\mu \\
\left(\mathrm{mg} \mathrm{L}^{-1}\right)\end{array}$ & $\begin{array}{c}\mathrm{LD} \\
\left(\mathrm{mg} \mathrm{L}^{-1}\right)\end{array}$ & $\begin{array}{c}\mathrm{LQ} \\
\left(\mathrm{mg} \mathrm{L}^{-1}\right)\end{array}$ \\
\hline EAA & $\mathrm{H}_{2} \mathrm{SO}_{4} / \mathrm{H}_{2} \mathrm{O}_{2}$ & $0,45 \pm 0,02$ & 0,04 & 0,20 \\
& $\mathrm{HNO}_{3} / \mathrm{H}_{2} \mathrm{O}_{2}$ & $0,43 \pm 0,05$ & 0,06 & 0,13 \\
Colorimetria & $\mathrm{H}_{2} \mathrm{SO}_{4} / \mathrm{H}_{2} \mathrm{O}_{2}$ & $0,44 \pm 0,01$ & 0,01 & 0,08 \\
& $\mathrm{HNO}_{3} / \mathrm{H}_{2} \mathrm{O}_{2}$ & $0,41 \pm 0,08$ & 0,01 & 0,08 \\
\hline
\end{tabular}


Por meio das estimativas dos desvios padrão $\mathrm{s}_{\mathrm{H} 2 \mathrm{SO} 4}$ e $\mathrm{s}_{\mathrm{HNO} 3}$ para EAA e UV-Vis é possível comparar os resultados obtidos para as duas metodologias para a determinação do metal, por meio do Teste $\mathrm{F}$ e do Teste $\mathrm{t}$ de Student, utilizados anteriormente para avaliar as metodologias de digestão.

Para as amostras digeridas com $\mathrm{H}_{2} \mathrm{SO}_{4} / \mathrm{H}_{2} \mathrm{O}_{2}$, o valor tabelado de $\mathrm{F}$ para 3 determinações corresponde a 4,26, e para amostras digeridas com $\mathrm{HNO}_{3} / \mathrm{H}_{2} \mathrm{O}_{2}$, o valor tabelado de $\mathrm{F}$ para 9 determinações corresponde a 19,38. Os valores de $\mathrm{F}$ calculados com digestão por $\mathrm{H}_{2} \mathrm{SO}_{4} / \mathrm{H}_{2} \mathrm{O}_{2}$ e por $\mathrm{HNO}_{3} / \mathrm{H}_{2} \mathrm{O}_{2}$ foram de 1,78 . Esses valores estão abaixo dos valores tabelados, indicando que os dois métodos podem ser utilizados para determinação de zinco com digestão de amostra por ambas as misturas.

$\mathrm{O}$ resultado obtido para t de Student com digestão por $\mathrm{H}_{2} \mathrm{SO}_{4} /$ $\mathrm{H}_{2} \mathrm{O}_{2}$ foi de 0,488 , e por digestão por $\mathrm{HNO}_{3} / \mathrm{H}_{2} \mathrm{O}_{2}, 0,971$. Sendo o valor tabelado para 10 graus de liberdade 2,228 , pode-se afirmar que as duas metodologias são equivalentes para determinação de zinco com digestão por $\mathrm{H}_{2} \mathrm{SO}_{4} / \mathrm{H}_{2} \mathrm{O}_{2}$ e $\mathrm{HNO}_{3} / \mathrm{H}_{2} \mathrm{O}_{2}$.

\section{CONCLUSÕES}

Por meio dos valores dos coeficientes de correlação (r) obtidos para as curvas por EAA e colorimetria, observa-se boa linearidade por ambos os métodos na faixa de concentração em estudo.

Pelos valores calculados de F, como teste de comparação dos dois métodos de digestão, conclui-se que este valor está dentro do valor tabelado, tanto nas determinações por EAA como por UV-Vis. Este fato é confirmado pelo teste t, o que significa que os métodos podem, possivelmente, ser utilizados para determinação de zinco.

Os valores de $\mathrm{F}$ calculados para comparação dos métodos indicam que ambos podem ser utilizados com bons resultados, já que os valores para os dois tipos de abertura de amostra encontraramse dentro dos valores tabelados. $\mathrm{O}$ mesmo pode ser visto pela aplicação do teste t.

Pode-se concluir que o método proposto para digestão de amostras de solo para determinação de zinco com $\mathrm{H}_{2} \mathrm{SO}_{4} / \mathrm{H}_{2} \mathrm{O}_{2}$ apresenta bons resultados quando comparados aos obtidos por $\mathrm{HNO}_{3} / \mathrm{H}_{2} \mathrm{O}_{2}$, uma mistura utilizada mais freqüentemente para esta finalidade.

Assim, a metodologia colorimétrica, juntamente com sua aparelhagem compacta, revelou possuir grande eficácia na determinação de zinco em amostra de solo, podendo ser aplicada em análise de rotina na agricultura.

\section{AGRADECIMENTOS}

Os autores agradecem à FAPESP (projeto 03/03700-1), ao CNPq e à CAPES pelo suporte financeiro.

\section{REFERÊNCIAS}

1. Trubetskoj, O. A.; Kudryavceva, L. Y.; Shirshova, L. T.; Soil Biol. Biochem. 1991, 23, 1179.

2. Kiehl, E. J.; Fertilizantes Orgânicos, Agronômica Ceres Ltda: Piracicaba, 1985.

3. Malavolta, E.; Fertilizantes e seu Impacto Ambiental: metais pesados, mitos, mistificação e fatos, Produquímica: São Paulo, 1994, p. 153.

4. Manahan, S. E.; Environmental Chemistry, Lewis Publishers: USA, 1994, p. $459-471$.

5. http://www.sna.agr.br/artigos/artitec-micronutrientes.htm, acessada em Abril 2005.

6. APHA; Standard Methods for the examination of water and waste water, $17^{\text {th }}$ ed., Washington: USA, 1989.

7. Alloway, B. J.; Heavy metals in soil., Blackie Academic and Professional: Glasgow, 1995.

8. Hesse, P. R. A.; Textbook of Soil Chemical Analysis, Chemical Publishing Co. Inc.: New York, 1972, p. 428.

9. Van Raij, B.; Quaggio, J. A.; Cantarela, H.; Ferreira, M. E.; Lopes, A. S.; Bataglia, O. C.; Análise Química de Solo para fins de fertilidade, Fundação Cargil: Campinas, 1987.

10. Sillampãã, M.; Vlek, P. L. G.; Fert. Res. 1995, 7, 151

11. Kalayci, M.; Torun, B.; Eker, S.; Aydin, M.; Ozturk, L.; Cakmak, I.; Field Crops Res. 1999, 63, 87.

12. Graham, R. D.; Welch, R. M.; Breeding for staple-food crops with high micronutrient density, Working Papers on agricultural Strategies for Micronutrients, n. 3, International Food Policy Institute: Washington DC, 1996.

13. Ohse, S.; Santos, O. S.; Morodim,V.; Manfron, P. A.; Rev. Fac. Zootec. Vet. Agro. 1998/99, 5/6, 35.

14. http://www.agroagenda.com.br/noticias/detalhe_ult_not.asp?noticia=15248, acessada em Abril 2005.

15. http://www.epa.gov/epaoswer/hazwaste/test/pdfs/3050b.pdf, acessada em Abril 2005.

16. http://www.epa.gov/epaoswer/hazwaste/test/pdfs/7000a.pdf, acessada em Abril 2005.

17. http://www.epa.gov/epaoswer/hazwaste/test/pdfs/6010b.pdf, acessada em Abril 2005.

18. Chapin, T. P.; Wanty, R. B.; Anal. Chim. Acta 2005, 543, 199.

19. Morozko, S. A.; Ivanov, V. M.; J. Anal. Chem. 1997, 52, 777.

20. The Handbook Odyssey DR/2500 Spectrophotometer: Procedure Manual, Loveland: HACH Company, 2001.

21. Instruction Manual - Digesdahlâ Digestion Apparatus Models 23130-20,21, HACH Company, 1999.

22. http://www.cnps.embrapa.br/search/pesqs/tema4/tema4.html, acessada em Janeiro 2004.

23. Alberguini, L. B. A.; Silva, L. C.; Rezende, M. O. O.; Quim. Nova 2003, $26,291$.

24. Alberguini, L. B. A.; Silva, L. C.; Rezende, M. O. O.; Tratamento de resíduos químicos: guia prático para a solução dos resíduos químicos em instituições de ensino superior, Rima: São Carlos, 2005.

25. Miller, J. C.; Miller, J. M. ; Statistics for analytical chemistry, $2^{\text {nd }}$ ed., Elis Horwood: Chichester, 1988.

26. Harris, D. C.; Análise Química Quantitativa, $5^{\text {a }}$ ed., LTC - Livros Técnicos e Científicos: Rio de Janeiro, 2001.

27. Baccan, N.; Química Analítica Quantitativa Elementar, $3^{\mathrm{a}}$ ed., Ed. Edgard Blücher: Campinas, 2001. 